\title{
WCCC 2019: the 25th World Computer Chess Championship
}

Article

Accepted Version

The ICGA WCCC 2019 report

Krabbenbos, J., van den Herik, J. and Haworth, G. (2020) WCCC 2019: the 25th World Computer Chess Championship. ICGA Journal, 41 (4). pp. 206-221. ISSN 1389-6911 doi: https://doi.org/10.3233/lCG-190125 Available at https://centaur.reading.ac.uk/85845/

It is advisable to refer to the publisher's version if you intend to cite from the work. See Guidance on citing.

Published version at: https://content.iospress.com/articles/icga-journal/icg 190125

To link to this article DOI: http://dx.doi.org/10.3233/ICG-190125

Publisher: The International Computer Games Association

All outputs in CentAUR are protected by Intellectual Property Rights law, including copyright law. Copyright and IPR is retained by the creators or other copyright holders. Terms and conditions for use of this material are defined in the End User Agreement.

\section{www.reading.ac.uk/centaur}

\section{CentAUR}

Central Archive at the University of Reading

Reading's research outputs online 


\title{
WCCC 2019: The $25^{\text {th }}$ World Computer Chess Championship
}

\author{
Jan Krabbenbos, Jaap van den Herik and Guy Haworth ${ }^{1}$ \\ Amersfoort, the Netherlands, Leiden, the Netherlands and Reading, UK
}

The 25th World Computer Chess Championship started on August 13, 2019. Six programs as in Table 1 participated in a double round robin tournament of ten rounds. The tournament took place at the Venetian in Macau, China and was organized by the ICGA. The venue was part of the $28^{\text {th }}$ International Joint Conference on Artificial Intelligence (IJCAI-19) who acted also as the main sponsor. The other important sponsor was Digital Game Technology (DGT) who provided the electronic chess boards. Chessbase donated an extra prize for the first position. The WCCC was held in conjunction with the 'WCSC' 9 $^{\text {th }}$ World Chess Software and 'WSCC' 2019 World Speed Chess Championships: all the moves are available (Krabbenbos et al., 2019a-2019c). The classical tempo of $1 \mathrm{~h} 30^{\prime}+15^{\prime \prime} / \mathrm{m}$ was used. Below, we provide the highlights of each game.

Table 1

The participants in WCCC 2019 (CPW, 2019) ${ }^{2}$

\begin{tabular}{|c|c|c|c|c|c|c|c|}
\hline id & Program & Author(s) & $\mathbf{C C}$ & Operator & Hardware & GHz & Cores \\
\hline $\mathrm{C}$ & CHIRON & Ubaldo Andrea Farina & IT & Ubaldo Andrea Farina & $2 \times$ Intel Platinum 8175 & 2.5 & 48 \\
\hline G & GIN & & $\mathrm{D}$ & & v2 & 2.8 & \\
\hline $\mathrm{J}$ & JONNY & Joha & $\mathrm{DE}$ & Johannes Zwanzger & eon E5-2630 & 2.4 & 1,200 \\
\hline K & KOMODO & $\begin{array}{c}\text { Don Dailey, Larry Kaufman } \\
\text { \& Mark Lefler }\end{array}$ & US & Erdogan Günes & $8 \times$ Intel Xeon Platinum 8168 & 2.7 & 128 \\
\hline $\mathrm{S}$ & & Stefan Meyer-Kahlen & $\mathrm{DE}$ & Stefan Meyer-Kahlen & 24 x Amazon Intel Xeon E5 & 2.6 & 48 \\
\hline $\mathrm{T}$ & THE BARON & Richard Pijl & NL & Richard Pijl & 2 x Intel Xeon E5-2697 & 2.6 & 28 \\
\hline
\end{tabular}

Round 1: CHIRON - GINKGO 1/2-1/2, SHREDDER - KOMODO 1/2-1/2, THE BARON - JONNY 0-1.

THE BARON started the official World Championship in a good mood and had prepared an opening with a frivolous aspect. The queenside castling of the white king was a signal for JONNY to attack vigorously. It brought its a-pawn to a3 and a majority of its pieces to the queenside. In the position of Fig. 1a, White had the choice between b3 and the move it chose, 16. Nxb5. The latter move is very speculative and led to a dynamic position.

White replied to 16.... Qb6 with 17. Nc3. White had hoped for some time to protect its king adequately and in order to give its knight some support, play continued 17. ... axb2 18. Bc4 Na6 19. Qxb2 Qa7 20. Bb3 0-0 21. Be1 Rfb8 22. Bg3 Rb7 23. e4 c5. But in fact the white position was entirely unstable. The continuation looked like a Bond movie where THE BARON found a clever defense each time. Nevertheless, experienced chess players felt the sequence of ingenious escapes would come to an end and indeed, that happened on move 30 when Black played 30.... Ncxa4, see Fig. 1b. At this moment, the white position collapsed. Black had computed the consequences deeply and saw that it

\footnotetext{
${ }^{1}$ Corresponding author: g.haworth@ reading.ac.uk

${ }^{2}$ Komodo did not use its Monte-Carlo tree-search mode: SHrEDDER did use some neural-net functionality.
} 
could complete the combination with a knight fork delivering check. That created a material difference in favour of Black and White resigned immediately.

SHREDDER - KOMODO began with the Semi Slav opening: many variants have previously been played in this championship. KOMODO tried to play with originality but could only bring imbalance to the position by sacrificing a pawn. Erdogan Günes: 'In my opinion Black had more than sufficient compensation.' For White, the sacrifice was a sign to take the pawn and to try to play as safely as possible, and this policy certainly succeeded. Black had to find ingenious moves to keep the fight going. KOMODO was excellent in performing this task but could never achieve more than the draw agreed on move 38 .
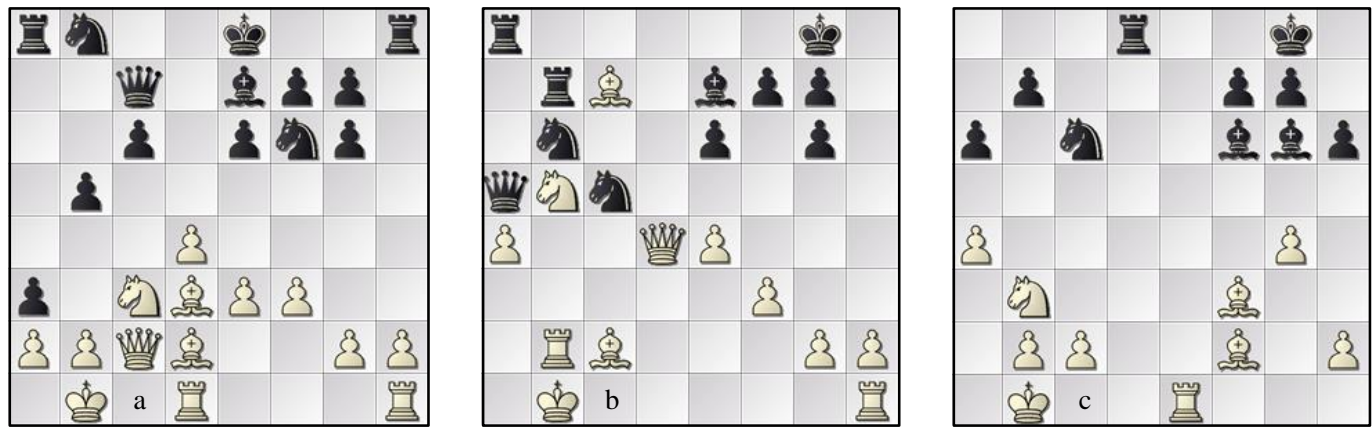

Fig. 1. Round 1: (a) THE BARON - JONNY position 16w and (b) 30b; (c) CHIRON - GINKGO 31w.

CHIRON and GINKGO produced a well-known variation of the Sicilian Najdorf. The play was accurate and never surprising. There was one position in which a far-reaching decision was taken by White, see Fig. 1c. White decided to give Black the advantage of the bishop pair and the disadvantage of a destroyed pawn structure. The idea did not give White the edge it had hoped for but the decision was well founded. During the remainder of the game, Black needed to protect its a- and c-pawns. White was never in an advantageous position but equally, it was not under threat. Subtle maneuvering led to a draw.

After R1: 1 JonNy 1; 2= CHIRON, GINKGO, KOMODO, SHREDDER 1²; 6 THE BARON 0.

Round 2: GINKGO - JONNY 1/2-1/2, KOMODO - THE BARON 1-0, CHIRON - SHREDDER 1/2-1/2.

The game GINKGO - JONNY was a high-level fight during which the opponents never saw more than a draw. Of course, they created small threats with some positional value. At one point, it looked as if White had been playing somewhat optimistically but in practice, there were no consequences either way for such a perspective. The game continued quietly and the draw was the clear result for players of equal strength.

KOMODO - THE BARON was a new challenge for THE BARON: it had to keep itself up and running against the current world champion. However, being world champion does not guarantee a won game. The authors and operators are well aware of their responsibilities: operator Erdogan also had a hand in KOMODO as the builder of its opening book. These responsibilities include taking some risks in order to obtain chances to win the game. In this particular game, White played 14. Rad1 and now Black may take the pawn on a2, see Fig. 2a. Somewhat later, Black was even able to win the exchange with 14. ... Bxa2 15. d4 Bb3 16. dxe5 Bxd1 17. Rxd1 Qc8 18. Nd5 0-0 19.Nh4 Kh8 20. Qg3 Nd8 21. Kh2 Ne6 22. f4 Qd8, see Fig. 2b. 
The difficulty which Black was facing is best expressed as 'Where do I place my knight?' What happened is that the knight could not find a safe place and was finally taken by White at the cost of a pawn and the exchange of a rook. But the two knights were far superior to the black rook in the resulting end game. The resignation was not unexpected. Computer programs had at that moment an evaluation of +8 although human beings still had to compute the lines - but every chess player understands that White is the winning side.
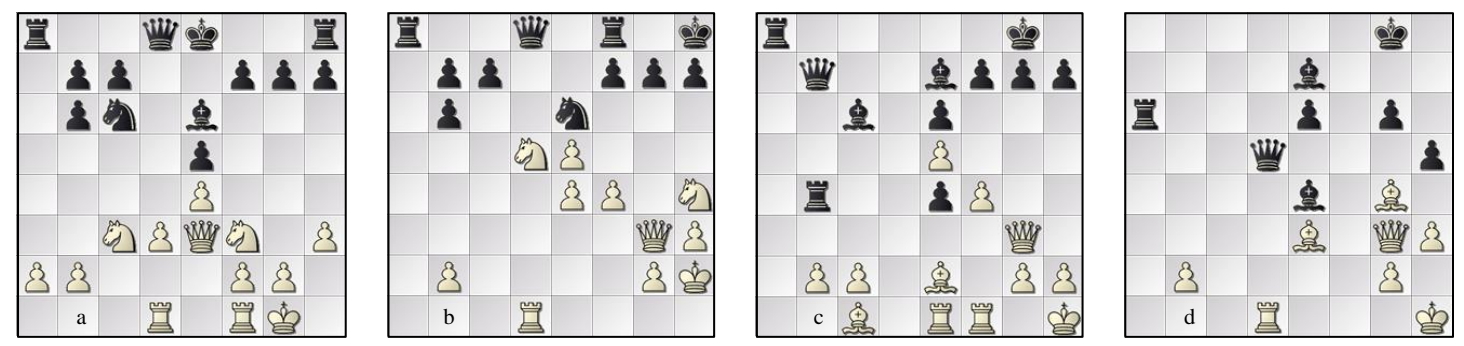

Fig. 2. Round 2: (a) KomOdo - THe BARON 14b and (b) 23w; (c) CHIRON - SHREDder 25w and (d) $37 \mathrm{~b}$.

The game CHIRON - SHREDDER started as a Scheveningen Sicilian. This is one of the most frequently played openings in the chess world and therefore discussing the opening could take pages or could be achieved by a reference to the literature. We do so because the first 24 moves were in the programs' opening books. Thereafter, the game was fascinating for us humans. If you replay the game, you will be impressed by the possibilities that emerged from the position on the board - a labyrinth of alternative and equally good positions. It will be a joy for human beings to analyze this game. When Ubaldo Andrea Farina was asked for his opinion of the game, he answered: 'It was a boring game. After the opening both programs agreed on a zero evaluation and throughout the game it remained a zero evaluation.'

When checking this opinion with Stefan Meyer-Kahlen, he stated: 'Yes, I fully agree with the zero evaluation remark and for myself, I did not understand what was happening. The possibilities were too numerous and too distinct from each other. Only a program can find the best lines and remain on the correct path among many moves.' For this reason, we illustrate this game with just two diagrams. We start with the departure from the opening book, Fig. 2c. White played 25. f5. There followed 25. ... exf5 26. Rxf5 e3 27. Rg1 g6 28. Rf4 Rxf4 29. Qxf4 Ra1 30. Qg3 Be4 31. e6 fxe6 32. Bg4 Qd5 33. Qh3 Ra6 34. Bxe3 Bxc2 35. Qg3 Be4 36. h3 h5 37. Rd1, Fig. 2d, 37. ... Bxg2+ 38. Kh2 Qb3 39. Bxh5 Be4 40. Bxg6 Qxb2+ 41. Rd2 Bd6 42. Qxd6 Rxd6 43. Rxb2 Bxg6 and a draw was agreed.

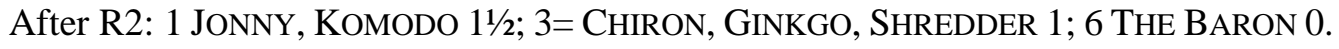

\section{Round 3: SHREDDER - GINKGO 1/2-1/2, THE BARON - CHIRON 0-1, JONNY - KOMODO 1/2-1/2.}

SHREDDER - GINKGO played a King's Indian variation. Apparently, the programs had respect for each other's playing strength. Occasionally, they played somewhat progressively if not aggressively but no action undertaken in this way resulted in a real threat. If you replay the game quickly, some small advantages may be apparent but deeper analysis will show that the game never offered strong, distinctive opportunities. It was an exciting game for those who seek after the truth but the programs agreed that the full point lay precisely between them: game drawn.

THE BARON played the Four Knights opening and managed to achieve a comfortable position. Still, comfortable positions are difficult to play because of their apparent ease: the hidden pitfalls may only be visible at deeper levels of the search tree. White had progressed its knight to d6 but in Fig. 3a, 23. 
Nf5 seems wise. However the program preferred 23. bxc4 and, to its surprise, Black took up the chance and reacted with the solid reply 23. ... Qxb1 24. Rxb1 Rxb1+ 25. Kh2 dxc4 and now we are at the crucial position of Fig. 3b. Obviously, 26. Bxc4 is impossible (26. ... Ng4+ 27. $\mathrm{Kh} 3 \mathrm{Rh} 1 \#)$ but there were two better alternatives to the move played in 26. g4 and 26. Bf3. As the game went, Black got the upper hand.

26. f3 Re1 27. Bxc4 Bxc4 28. Nxc4 Rdd1 29. g4 h5 30. Kg3 hxg4. Although this position has an evaluation of only -1.6 against White, the game can be seen as the start of playing a lost situation as well as possible. White did that but defeat could only be delayed. The following moves show this clearly: 31. Qc7 Kh7 32. Kf2 Nh5 33. fxg4 Rf1+ 34. Ke3 Nf4 35. Qa5 Nxg2+ 36. Ke2 Nf4+ 37. Ke3 Rde1+ 38. Kd2 Re2+ 39. Kc3 Rff2 40. Kb3 Rxc2 41. Qf5+ Ng6 42. Qh5+ Kg8 43. Qa5 Nf8 44. Qxa7 Rce2 45. Kb4 Rf4 46. Kb5 Rxg4 47. a4 Rg5+ 48. Ka6 Re6+ 49. Nb6 Rge5. In the last position, the evaluation is not that obvious for humans but it is for computers who see an evaluation of -8.0 against White. No wonder White resigned.

Some games are a pleasure to replay since you see many nice and interesting variations tumbling from the board. Other games are a challenge for the spectators since they wait for exciting manoeuvers or for surprising attacks. In the game JONNY against KOMODO we saw chess of the highest calibre. Two very well documented programs equipped with excellent search functions were fighting each other. However, their fight was subtle, repetitive and not challenging the brains of the human replayer. It was chess, chess and once more chess. The play was of high standard but it all remained in the draw area of \pm 0.01 .

\section{After R3: 1= CHIRON, JONNY, KOMODO 2; 4= GINKGO, SHREDDER 1느; 6 THE BARON 0.}
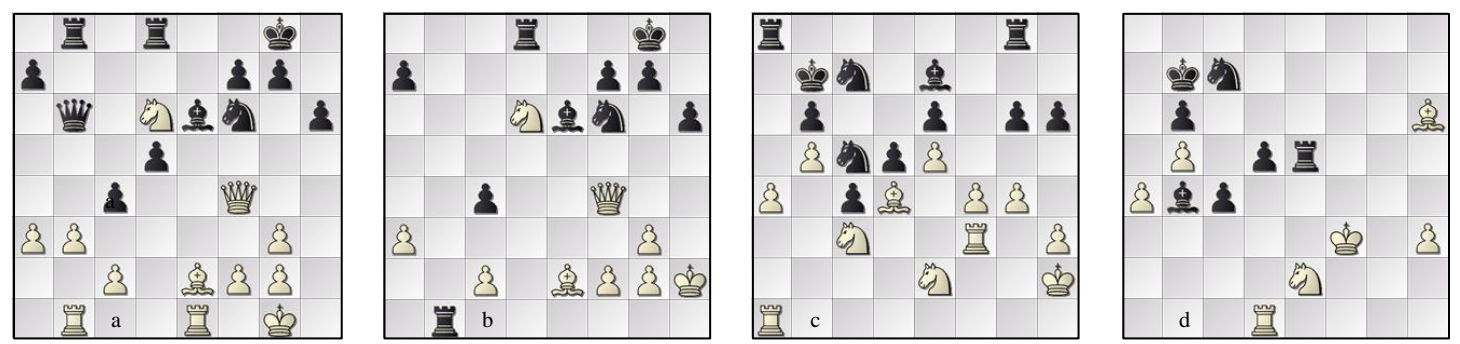

Fig. 3. (a) Round 3, THe BARON - ChiRon 23w and (b) 26w; (c) Round 4, GinKGO - Komodo 52w and (d) 65b.

\section{Round 4: GINKGO - KOMODO 0-1, CHIRON - JONNY 1/2-1/2, THE BARON - SHREDDER 0-1.}

The preparation of world champion KOMODO has been excellent and is also to be characterized as trying to find the limits of what is just possible to start a real fight. Current opening theory is worked out almost up to the end with equal play as the verdict. Then it is the task of the computers to prove that this statement is true, which in many cases happens to be the case. Interestingly there are also positions which are less frequently played and where the outcome has a wider interval of uncertainty. Erdogan Günes together with his team mates from KOMODO have tried to specialize in incorporating special variations which are sidelines from the main variations. These variations may give support to original play in double-edged positions. Sometimes it is very hard to see how the play works out and how KOMODO can make use of the risks it has taken. In this game, it looks like the infrequently played opening is the start of a fight but White played very accurately and precisely. Therefore, it took a long time for KOMODO's main ideas to come to the surface. Having said this, the reader should keep in mind the importance of the center pawns even if they are blocked. We refrain from analyzing the first 51 moves and start with move 52, see Fig. 3c. 
At this moment White was challenged to play the breakthrough move 52. f5. Looking at the pawn structure we see that White has a majority of pawns at the queenside (a-b vs b) and a majority on the kingside (f-g-h vs g-h). The black pawns in the center seem to be blocked and White believes that is possible to keep that blocking for the rest of the game. It turns out not to be the case and it is to the credit of KOMODO to have recognized this opportunity. 52... Nb3 53. Rd1 exf5 54. Bf2 Bg5 55. gxf5 gxf5 56. Nd4 Nxd4 57. Rxd4 Be7 58. Rd1 Rad8 59. Rxf5 Rgf8 60. Rxf8 Rxf8 61. Be3 Rd8 62. Kg3 Bb4 63. Ne2 Re8 64. Kf3 Rxe5 65. Bxh6 see Fig. 3d.

This position was aimed at by KOMODO. The two passed pawns in the center are compensated for by a worthless h-pawn which needs much support. Black now has to develop a winning plan. KOMODO does so adequately in the following line: 65 .... Ne6 66. h4 Bc5 67. Bd2 d4 68. Nf4 Nxf4 69. Bxf4 Re7 70. h5 c3 71. h6 Rf7 72. Rg1 Bf8 73. Rh1 d3 74. Ke4 d2 75. h7 Bg7 76. Bxd2 Re7+ 77. Kd3 Rd7+ 78. Kc2 Rxd2+ 79. Kc1 Bh8 80. Rh6 Rf2 81. Rh4 Rg2 82. Rf4 Rg7 0-1.

From the beginning both players, CHIRON and JONNY, showed equal strength. They took an original path in the opening but nothing special happened until move 19 when CHIRON saw the opportunity of sacrificing the exchange, see Fig. 4a.

19. Rxf6 gxf6 20. Bf4. Black saw the dangers which it had to counter quiet clearly and decided to reimburse White by immediately returning the advantage of the exchange. 20. ... Nc6 21. Bxe5 Nxe5 22. exd5 Re8. Now the position is understandable by the programs and they agreed that it is an equal game. Nevertheless they continued to play as follows: 23.Qf1 Qc8 24.Rc2 Ng4 25.Re2 Kf8 26. Rxe8+ Qxe8 27. Nf5 Ne3 28. Nxe3 Qxe3+ 29. Kg2 Qd2+ 30. Qf2 Qxd3 31. Qc5+ Kg7. They did so to test the opponent's alertness. Having seen that both sides had control on the dynamics of the positions, they completed the game by 32. d6 Qe2+ 33. Kh1 and a draw was agreed.

THE BARON - SHREDDER was a game of manoeuvering. THE BARON was well prepared and in the first instance, moved very well. Still, in the complications of the middle game, Black was able to equalize the position completely and was thereafter fighting for the upper hand. This required a playing forwards and backwards and a kind of seducing of White to place its pieces on the near optimal squares. Some of the near-optimal moves led to a position in which Black obtained more space and was even able to penetrate the white position. However penetrating did not mean gaining material or creating a mating network. In a difficult position, White made a mistake and Black achieved a clear winning advantage, see Fig. $4 \mathrm{~b}$.
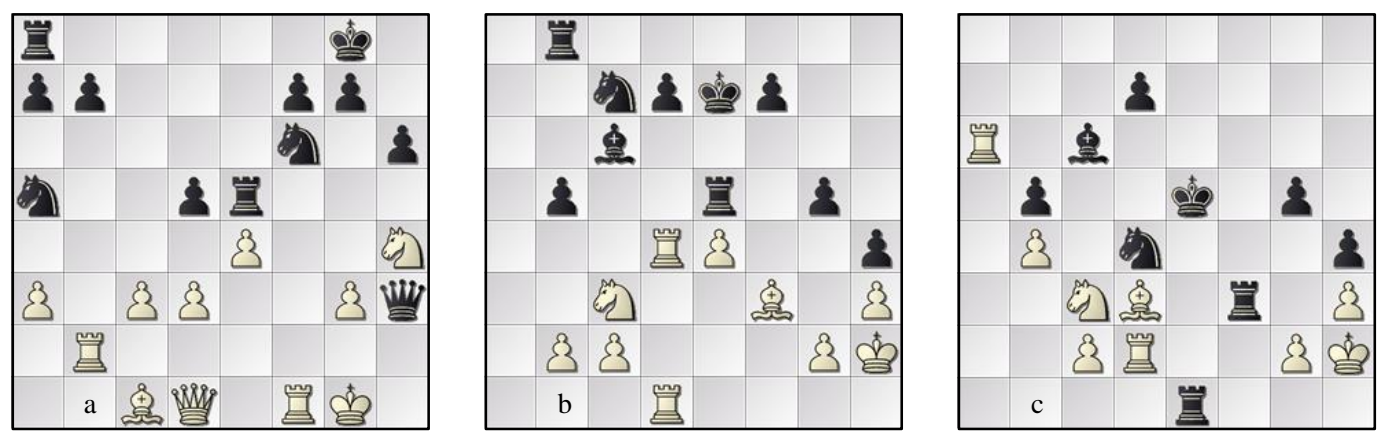

Fig. 4. Round 4: (a) CHIRON - JONNY 19w; (b) THE BARON - SHREDDER 26w and (c) 44w.

White played 26. Rb4 instead of 26. b4. After Rb4, the evaluation function went down and by strong play from Black's side it went down further in the next ten moves. In Fig. 4c, the position was already 
lost and therefore White's move Rd1 cannot be considered a blunder. But after this move, Black undoubtedly concluded this game convincingly.

36. Rd1 Rf8 37. Rd2 Re1 38. Ra6 Rf4 39. Na2 Rf3 40. b4+ Kd5 41. Be2 Rf4 42. Bd3 Ke5 43. Nc3 Rf3 44. Rd1 Rxd1 45. Nxd1 Rg3 46. Bf1 Nxc2 47. Ra2 Nxb4 48. Re2+ Kd4 49. Rd2+ Kc5 50. Nf2 Ra3 51. Rb2 d5 52. Rb1 d4 53. Rd1 Rc3 54. Kg1 Nc2 55. Rb1 Ne3 56. Nd3+ Kb6 57. Ne5 Nxf1 58. Kxf1 Re2: White gave up.

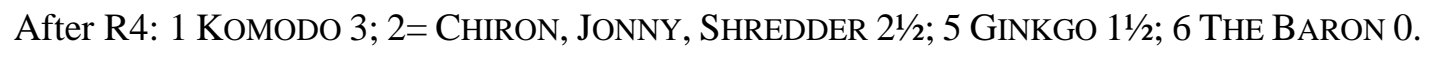

Round 5: THE BARON - GINKGO 1/2-1/2, JONNY - SHREDDER 1/2-1/2, KOMODO - CHIRON 1/2-1/2.

In this round, THE BARON showed that it had the robust character needed by chess players - not being depressed by the four zeros it had to accept so far. It played GINKGO and saw that its efforts produced fruits and small successes. A small success is defined here as reaching a position with chances for a win. The opening went well for THE BARON. In the middlegame the programs seemed to play on equal footing. It was not easy since the positional rather than tactical complications were difficult to assess. An example of these considerations is given in Fig. 5a.

White had sacrificed a pawn and in exchange had achieved positional pressure along the b-file, occupation of the important square $\mathrm{d} 5$ by a knight, a blocked d-pawn and an advantageous situation on the kingside. Black's threat was Bxf4 followed by g5 but White was on move and prevented this variation with 29. h4. The game then continued by 29. ... Bg7 30. Kh2 Rfc8 31. Qe2 Re8 32. Qg2 Qf7 33. Rb6: White was increasing the pressure on the black position. That was a good goal in itself but not sufficient for a win so after 15 more moves, a draw was agreed.

JONNY and SHREDDER played an intriguing game in which both players clearly intended to defeat their opponent. They did so with modest means that look quite aggressive on the board. Structure, activity and king safety rather than the pawn count were the most important considerations. According to these three measures the game went up and down. First, White had the initiative and Black was the architect of the kingside, progressing its pawns. On the queenside, White sacrificed two pawns for open squares, open lines and combinatorial possibilities. However, the reply by Black made White cautious on the kingside. Around move 24, the picture was clear, see Fig. 5b.

It is not easy to describe but White's opportunities are on the queen side. Black's situation on the kingside is easier to talk about. The main question is 'how dangerous is the black attack?' Black played 24. ... 0-0-0 which was followed by 25 . Ne5 Bxe5 26. dxe5 Rgg8 27. Rd6, see Fig. 5c.
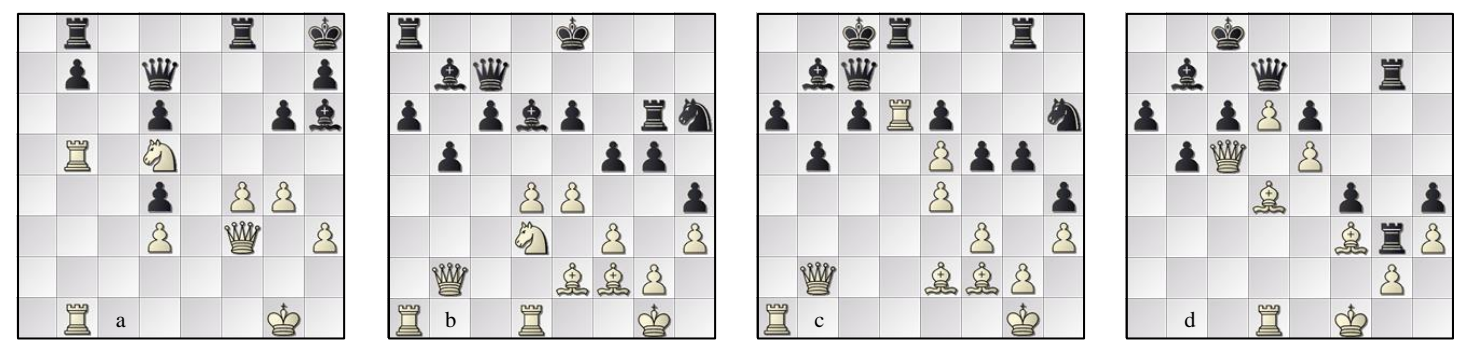

Fig. 5. Round 5: (a) THE BARON - GINKGO 29w; (b) JONNY - SHREDDER 24b, (c) $27 \mathrm{~b}$ and (d) 42b.

By this move, White kept the battle going since Rxd6, exd6 and Qxd6 are followed by Qf6. It meant that Black had no time to execute its plans on the kingside. Attention should be given to the intruder. 27. ... Rge8 28. Rad1 Nf7 29. Bc5 This implies the sacrifice of the exchange. Black must accept and 
thereafter defend its position: 29. ... Nxd6 30. exd6 Qh7 31. e5 Rd7 32. Re1 Rg8 33. Qa2 Qg6 34. Bd1 Rf7 35. f4 Rfg7. Opening the g-file by gxf4 is not an option for Black since the white bishop on $\mathrm{f} 3$ is a superb defender. Therefore, the tension should stay in the pawn formation. 36. Bf3 Qf7 37. fxg5 Rxg5 38. Kf1 R8g7 39. Rd1 Qd7 40. Qa3 f4 41. Bd4 Rg3 42. Qc5, see Fig. 5d.

42. ... Rxf3+. Black returns the exchange sacrifice to White in order to have an open file at its disposal for counterplay since it is clear that the black bishop on b7 is not a participant in the battle. The game continued 43. gxf3 Qd8 44. Bf2 Kd7 45. Be1. Subsequently Black succeeded in freeing its blocked queenside pawns by sacrificing its a- and b- pawns. 45. ... a5 46. Qa7 Qa8 47. Qb6 Qc8 48. Rc1 b4 49. Qxa5 b3 50. Kf2 Ba6 51. Qb6 Qb7 52. Qxb7+ Bxb7 53. Rc3 c5 54. Rxb3. The resulting endgame was then materially in balance and the bishops of opposite colour guaranteed the draw.

The game KOMODO - CHIRON was of an experimental nature which started as early as the second move. After 1. d4, White played 2. Nd2 so opening theory was written in this game. But that is not the main point of interest. First we saw how top programs were able to handle closed positions by repeatedly placing their pieces on very well chosen squares. Knowing each other's strategy, they anticipated the moves of their opponent. The result was a boring game from both sides. Nobody would have been surprised if the players had at that moment agreed a draw. However both preferred to show more of their capabilities. On move 52, Black opened the position, see Fig. 6 a.

The play continued as follows $52 . .$. gxf4 53. gxf4 Qh5 54. Bh1 Rg4 55. Rg2 Rh4 56. Qe3 Rgg4 Then on move 57, there was a change in the material balance when Black managed to win White's queen at the cost of two rooks. 57. Rf2 Rxh3+ 58. Qxh3 Rh4 59. Bg2 Rxh3+ 60. Bxh3. Now the question is 'what is of more value, two rooks or a queen in an almost closed position?' 60. . ... Kh8 61. Rff1 Kg7 62. Re3 Kf8 63. Kg3 Ke7 64. Rh1 Kf7 65. Kg2 Kf8 66. Rhe1 Qh4 67. Rf1 Kg7 68. Rg3+ Kf7 69. Re3 Ke8 70. Rg3 Bd7 71. Rg7, Fig. 6b. For eleven moves, there was no progress then White saw the opportunity to penetrate the black position with a rook. Black reacted adequately by pushing its pawn into the white position: 71.... e3. The game continued 72. $\operatorname{Rg} 3$ e2 73. $\operatorname{Re} 1$ Qxf4 74. Rxe2 Be6 75. Rg6 Kf7 76. Rf6+ Ke7 77. Rf2 Qe4+ 78. Rf3 Qxd4 79. Bxf5 Bxf5 80. R3xf5 Qc3 81. Rxc6, all more or less forced and leading to the position of Fig. 6c.

The once closed position was now fully open. The question now is 'who will come out on top?' Since it looked as if Black had excellent opportunities, White resorted in Fig. $6 \mathrm{c}$ to the perpetual check tactic: 81. ... Qxa3 82. Rcf6 Qxb4 83. Rf7+ Kd8 84. Rf8+ Kd7 85. R8f7+ Ke6 86. R7f6+ Kd7 87. Rf7+ p87b, Fig. 6d, Ke6 88. R7f6+ Kd7 89. Rf7+ p87b, a 3x-repetition draw.

After R5: 1 KOMODO 31²; 2= CHIRON, JONNY, SHREDDER 3; 5 GINKGO 2; 6 THE BARON 1⁄2.
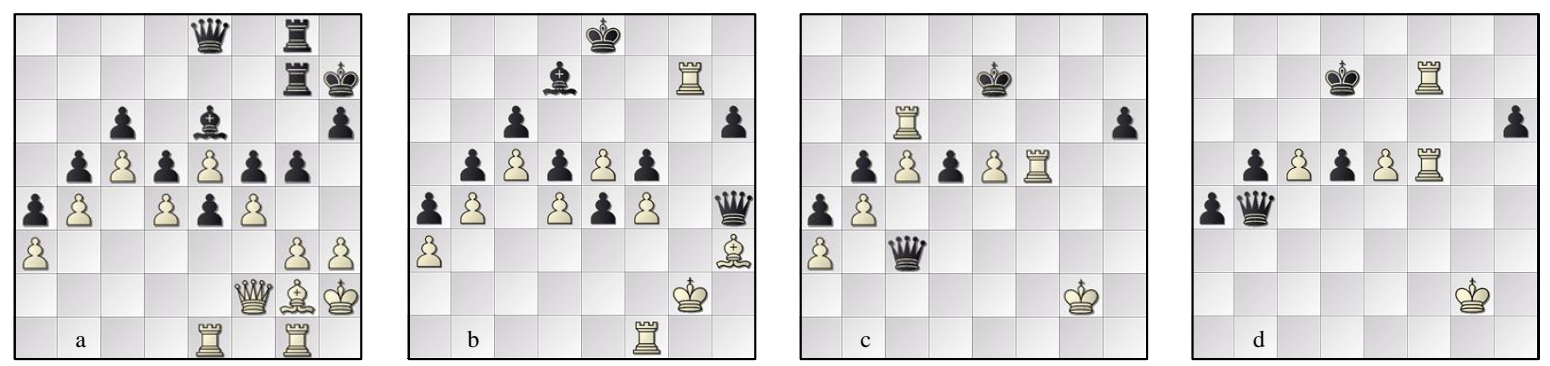

Fig. 6. Round 5: KOMODO - CHIRON (a) 52b, (b) 71b, (c) 81 b and (d) 87b/89b. 


\section{Round 6: GINKGO - CHIRON 1/2-1/2, KOMODO - SHREDDER 1-0, JONNY - THE BARON 0-1.}

'Remarkable, remarkable, remarkable' is the best characterization of this WCCC's sixth round. Many well-known WCCC games have been played but the game JONNY versus THE BARON ranks \#1 in remarkability - not for the fact that THE BARON won the game but for the way it achieved this performance. THE BARON had clearly been the weakest program of the six participants. It has a rating of approximately 2840 . This means that it ranks amongst the best humans but is not a super champion like the other programs. In the WCSC round 9, THE BARON had achieved a draw against JONNY with the same opening variation. It is an English opening with 8. ... g5, see Fig. 7a.

For THE BARON it was a book move but JONNY was out of book. In the WCSC game they played 9. d3 g4 10. Nd2 h5 11. b4 h4 12. Nc4. Now, JONNY played 12. b5, see Fig. 7b. The different move was caused by the change of platform. 12. Nc4 was played on the Lenovo laptop uniform platform. The cluster which now served as the platform produced 12. b5 as the best move. We note that JONNY had not explicitly adapted its opening book following the WCSC round 9 game.

The continuation was as follows: 12 .... Nd4 13. a4 hxg3 14. hxg3 a5 15. bxa6 Rxa6. This recapture is the point of the ideas behind this line of play. The a8-rook is brought into play via a6 to be active on the h-file. It is a really original idea in this variation. The play continued with $16 . \mathbf{a 5}$ Bb4 17. Nde4 Be7 18. Bb2 Nd7 19. Nd5 f5 20. Nd2 Rah6, see Fig. 7c.

Although there is no direct threat, the black rooks are enormously powerful and thus a continuous threat to the king. White was to become active on the queenside with its scope extending to the black king. This strategy led to a very remarkable development.

21. Bxd4 exd4 22. Qa4 Bg5 23. Rfc1 - the consequent follow-up from White's plan on the queenside but there is more to come: 23. ... c6 24. a6 Bxd2, see Fig. 7d. We are grateful for the variations given to us by Johannes Zwanzger. He provided us with insights into the lines of reasoning and also with the fact that he had detected a cluster design error which affected the computation of move 87 where JONNY played 87. Qa5 instead of the Qc5 which would have secured the draw.

Taking the knight is dangerous since it gives White an opportunity to continue the a-pawn's run to the promotion square. One wonders how the evaluation functions of both programs worked at this moment in this situation. 25. a7 Bxc1 26. a8=Q Bb2. White now has two possibilities - (a) 27. Rb1 and (b) 27.Qb4.
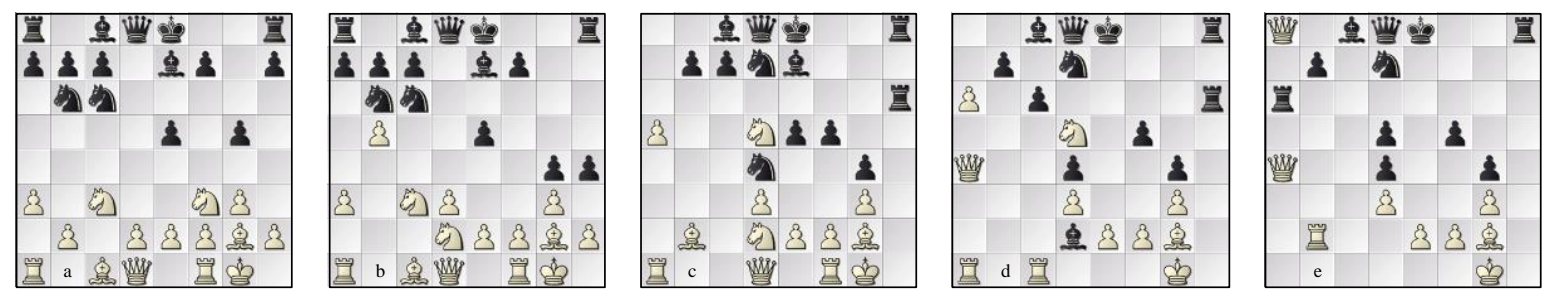

Fig. 7. Round 6: JONNY - THE BARON (a) 9w, (b) 12b, (c) 21w, (d) 25w and (e) sideline 'a', 29w.

We explore option (a): 27. Rb1 cxd5 28.Rxb2 and now Black has the wonderful defense of returning the rook on h6 to a6 leading to a won position, see the remarkable Fig. 7e.

We now explore line (b) which was actually played in the game. With two queens, all kinds of combinations are possible. This is a sharp line. White took the bishop c8, threatening mate on e7. 
After this manoeuver, the capture of the bishop on a1 is guaranteed. 27. ... Bxa1 28. Qxc8 cxd5 29. Qxd8+ Kxd8 30. Qa5+ Ke7 31. Qxa1, see Fig. 8a.

The material inequality, Q-vs-RR, B-vs-N, led to a difficult evaluation. We surmise that the situation is roughly equal. The question now is "What are the best positions for the black rooks currently on the h-file?" You will see that they are highly mobile and will be transferred to the first rank. 31. ... Rc8 32. Qxd4 Rc1+ 33. Bf1 Rhc6 34. Kg2 Rd1 35. Qg7+ Kd6 36. Qg6+ Kc7 37. Qxf5 Rec1 and now we are at Fig. 8b.

In a few moves the rooks found a new attacking position, now threatening the white king even more than before. The least threat is gaining a piece. White continued as follows: 38. Qf4+ Kc6 39. Qa4+ b5 40. Qxg4 Nb6 41. Qe6+ Kb7 42. Qe7+ Ka6 43. Qa3+ Na4 44. Qd6+ Ka5 45. Qxd5, Fig. 8c.
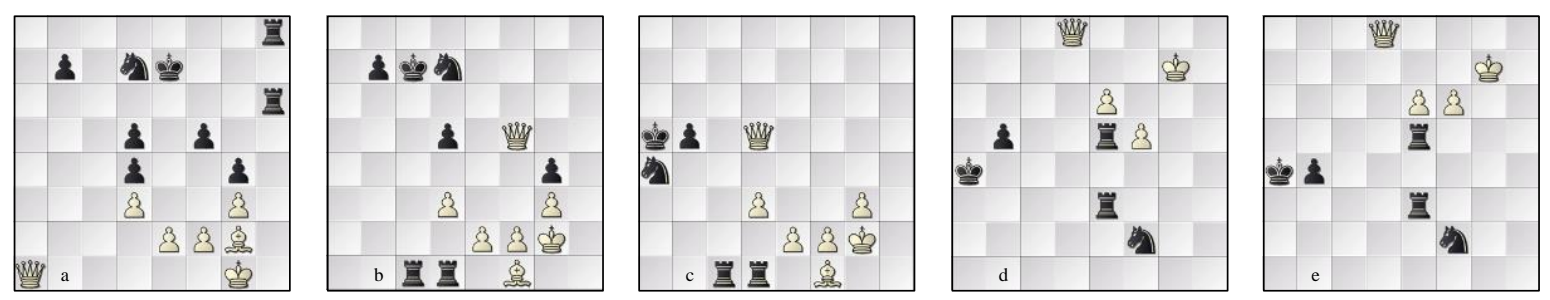

Fig. 8. Round 6: JONNY - THE BARON (a) 31b, (b) 38w, (c) 45b, (d) 57b and (e) 58b.

Finally, Black found the time to capture the white bishop. Now a new problem for the evaluation function arose: what is the value of the four pawns and what is the task of the black b-pawn with respect to Black's winning chances? The general thought was that the position is still a draw. However, the situation remained remarkable. White's task was to get the pawns running to their promotion squares. The prevailing question is which pawns should advance and which pawns should be given to Black? In the following, White's decisions are clear.

45. ... Rxf1 46. e4 Nc5 47. e5 Rfe1 48. f4 Rc3 49. f5 Re2+ 50. Kh3 Nxd3 51. Qd8+ Ka4 52. e6 Nf2+ 53. Kh4 Re5 54. Kg5 Rce3 55. Kg6 Rxg3+ 56. Kf6 Rge3 57. Kg7, Fig. 8d.

The e- and f-pawn lead the advance and are now close to their promotion squares. The b-pawn has not been given any attention or time to progress. Black has been too busy capturing white pawns and arranging the positions of its pieces in order to stop the white pawns running further. $\mathbf{5 7} . \mathbf{K g 7}$ and finally b4 58. f6, see Fig. 8e.

Here, Black has at least four moves which evaluate to a draw. They are 58. ... Rxe6, 58... Nd3, 58. ... Nh3 and 58. ... Ne4 as chosen. The game continued 59. Qd1+ Kb5 60. Qf1+ Kc6 61. Qa6+ Kd5 62. e7 Nc5 63. Qa8+ Kc4 64. Qg8+ Kc3 65. Qf8 Ne6+ 66. Kf7 Kc4 67. Qg8 Nf4 68. Qc8+ Kb3 69. Qa8 R3e4 70. Qa1 Re6 71. Qb1+ Kc3 72. Qa1+ Kc4 73. Qc1+ Kb5 74. Qf1+ Kc6 75. Qa1 Kb5 76. Qa2 - arbitrarily selected as it leads to a draw. JONNY could also have played 76. Qf1 repeating a previous position which is also evaluated as a draw. 76. ... R6e5 77. Qb3 Kb6 78. Qg3 Kc6 79. Qg8 Re6 80. Qc8+ Kb5 81. Qd7+ Kc4 82. Qc7+ Kd3 83. Qc5, Fig. 9a.

This move gives Black the opportunity to let its pawn step forward. The game is still evaluated as a draw but both players have to play accurately and diligently. We are soon arriving at the point where White missed the correct continuation as mentioned above. 83. ... b3 84. Qb5+ Kc3 85. Qc5+ Kd3 86. Qb5+ Kc3 87. Qa5+?, Fig. 9b.

Instead of repeating the position by playing 87. Qc5, White chose to play 87. Qa5+. JONNY has chosen once more not to repeat position, as with its 76. Qa2/Qf1 decision. There it did not make any 
difference but here it is a real error: Qa5+ does not lead to a draw. 87. ... Kb2 88. Qd2+ Kb1 89. Qc3, see Fig. 9c. At this moment, Black can exploit the mistake of Qa5+ by advancing the pawn again. 89. ... b2 90. Qb3 R6e5 91. Ke8 Re1 92. Kf7 R5e4 93. Qa3 R1e3 94. Qa5 Nd3 95. Kg8 Kc2 96. Qc7+ Kb3 97. f7, Fig. 9d.
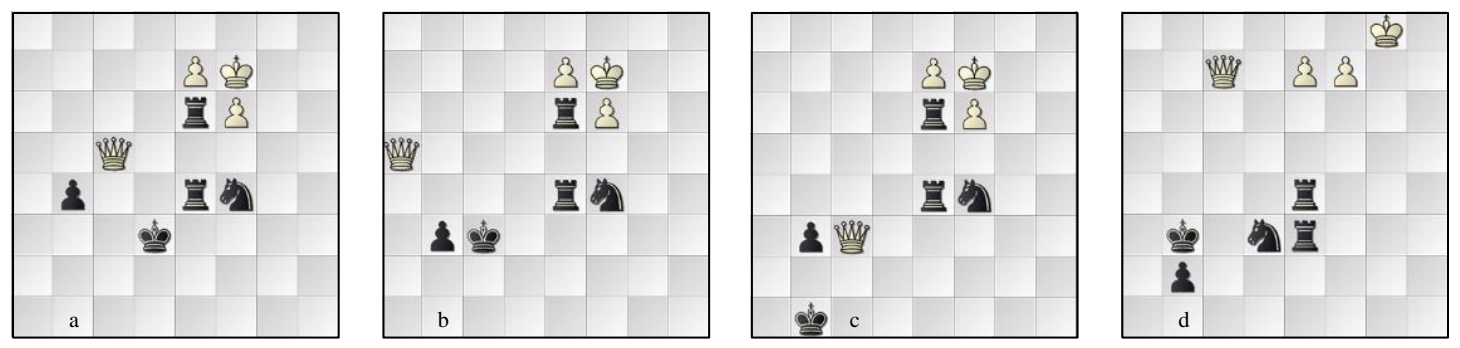

Fig. 9. Round 6: JoNNY - THE BARON (a) 83b, (b) 87b, (c) 89b and (d) 97b.

Now three pawns are candidates for promotion. Black has the move and plays $\mathbf{9 7} . . . \mathbf{b} \mathbf{b}=\mathbf{Q}$. White gives check and promotes the e-pawn but Black already has a winning score. 98. Qb8+ Nb4 99. e8=Q Qg1+ 100. Kf8 Qh1 'mate in 19 moves' 101. Qxb4+ Kxb4 102. Qb8+ Kc4 103. Qc7+ Kd3 104. Qd6+ Ke2 105. Qf6 Rb3 106. Qa6+ Rd3 107. Qa2+ Kf3 and White resigned, in fact only four moves short of mate.

GINKGO - CHIRON was a game between two players with similar superhuman strength. White managed to win a pawn but Black had seen well into the future. In the right positions, it developed pressure on the weak spots and that resulted in the regain of the pawn, after which a draw was inevitable.

KOMODO - SHREDDER started with an out-of-book opening 1. d4 2. Nd2. Here we again see Erdogan's hand in the opening book: play to your own strengths. For the average chess player, the development of both sides looked smooth. It seemed that both players reached a satisfying position. However, the deeper question was "Does Black have sufficient counter play for White's small strategically placed anchor points such as the pawn on h5?" SHREDDER followed the general line of being active in the center by playing 16.... f6, see Fig. 10a. In the game, SHREDDER had high hopes of finding sufficient counterplay by penetrating the white position with its e-pawn after 17. Nh4 Be4 18. Qd2 e5 19. Rfe1 Qd7 20. Rad1 Qe6 21. a3 Bxd3 22. Qxd3 e4 23. Qb5 f5 24. c4 Rfd8 25. cxd5 Nxd5 26. f3 Nce7 27. Qb3 Kh8 28. fxe4 fxe4 29. Qg3 e3 30. Nf3 Rf8 31. Ne5 Rf2, see Fig. 10b.
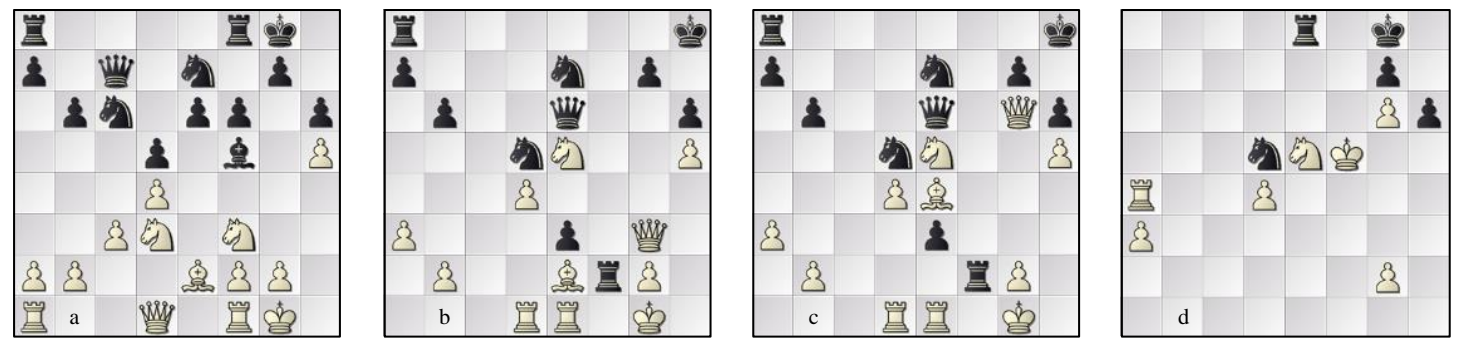

Fig. 10. Round 6: KoMOdO - SHREDDER (a) 17w, (b) 32w, (c) 34b and (d) 67b.

Nevertheless, Komodo had computed and evaluated the position very well. At the moment that Black believed it had sufficient counterplay, White strategically played the white bishop from e 2 via 
d3 to e4. This square was an excellent position for the bishop since from there it defended g2 and supported the queen's mating attack on g6. It transpired that all Black's hopes were in vain because KOMODO's combination of attack and defense was superior to SHREDDER's play. A convincing variation after 34. Qg6 see Fig. 10c here is 34. ... Nxg6 35.Nxg6 Kg8 (or 35. ... Kh7 36. Nf8++) 36.Bxd5 Qxd5 37.Ne7+. Black saw all threats and played 34. ... Qg8. On move 44, Black managed to exchange the queens on 66 but in the resulting endgame the white pawn on g6 kept the black king hostage in the corner. White regrouped its pieces and transformed the position gradually into the clearly won position of Fig. 10d.

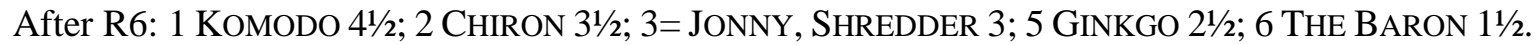

\section{Round 7: JONNY - GINKGO 1/2-1/2, THE BARON - KOMODO 0-1, SHREDDER - CHIRON 1/2-1/2.}

KOMODO continued to build up its own plans right from the opening position. The first moves were 1. e4 d6 2. d4 Nf6 3. Nc3 g6 4. Be3 c6 5. h3 b5 6. e5 b4 7. exf6 bxc3 8. fxe7 Qxe7 9. bxc3 Bh6 10. Qe2 Ba6 11. Qf3 f5 12. Be2 Bxe2 13. Nxe2 Bxe3 14. fxe3 0-0 15. 0-0. See Fig. 11a.

The weak squares on e4 and 44 are characteristics of the position. For the fixation of these squares, Black sacrifices another pawn by playing 15. ... Nd7. After 16. Qxc6 Nf6 17. Qc4+ d5 18. Qd3, Black had its target position and started to manoeuver. On move 35 the position shown in Fig. 11b was on the board.

Black is really satisfied. The pawn on a6 is not the main issue but in this phase it played an important role. The capturing of it was anticipated by 35. ... Nf2 36. Qd2 Rxa6 37. Rgb1 Ne4 38. Qe1 Rac6 39. R1b3 a6 40. Rb8 Qe7 41. R8b6 a5 42. Rxc6 Rxc6 43. Rb5 a4 44. Ra5 (44. Rxd5? Qa7 leads to a big advantage for Black). Thereafter White was able to eliminate the passed a-pawn. However the weak squares c4, e4 and g4 still remained easily accessible for the black pieces. After $63 . . . \mathrm{Ne} 4$ we were at Fig. 11c.

White now played 64. Ne6+ Kf7 65. Nxc7. It is remarkable to see how powerful the queen and black knight are. They outplayed White completely: 65. ... Ng5+ 66. Kh2 Nf3+ 67. Kh3 g5 68. hxg5 Nxg5+ 69. Kh2 Qh5+ 70. Kg1 Nh3+ and, as the queen must fall, White resigned.
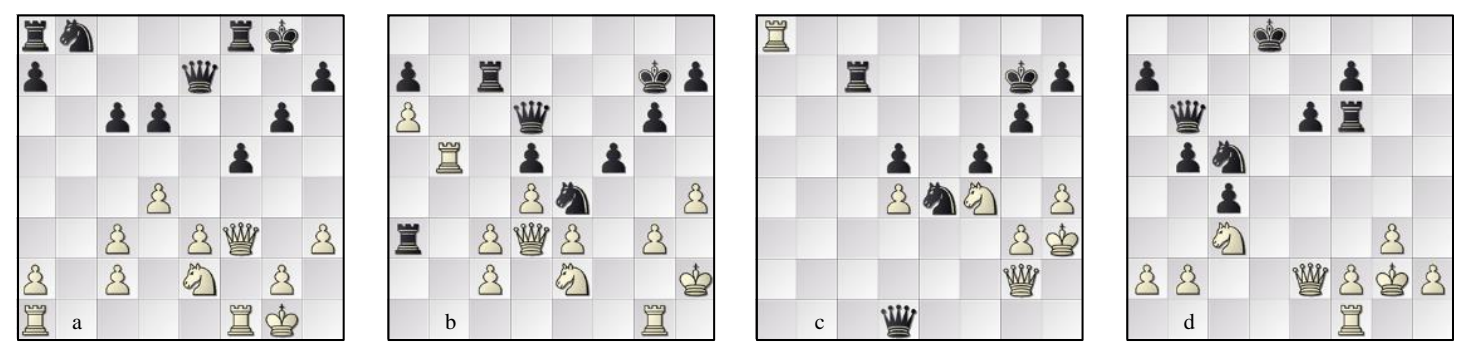

Fig. 11. Round 7: THE BARON - KOMOdo (a) 15b, (b) 35b, and 64w; (d) JONNY - GiNKGO 21 w.

JONNY - GINKGO played a game that followed both their opening books up to move 21 see Fig. 11d. For human beings, the game from here could have been exciting because there were many intricacies. However, for computer programs with grandmaster strength, it was easy to escape the tricks in the position. And so they moved smoothly to a drawn position after 21. Qe5 Qc6+ 22. f3 Ke7 23. a3 a5 24. Qb8 Nd3 25. h4 Rf5 26. Qa7+ Qd7 27. Qb6 b4 28. axb4 axb4 29. Ne4 Rb5 30. Qe3 Ra5 31. Ra1 Rxa1 32. Qg5+ Kf8 33. Qh6+ Ke7 34. Qg5+ Kf8, 1/2-1/2. 
The game SHREDDER - CHIRON started with a Semi-Slav. Although both sides had left the opening book around move ten, they both played solidly without creating difficult positions for their opponent. Accordingly, with both sides respecting the other's playing strength, the game ended after 51 moves in a draw.

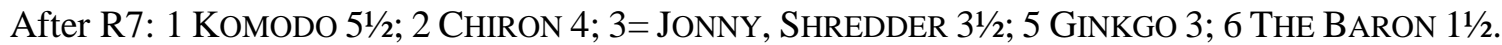

Round 8: GINKGO - SHREDDER 1/2-1/2, CHIRON - THE BARON 1-0, KOMODO - JONNY 1/2-1/2.

CHIRON - THE BARON is an example of a game between two players with different playing strengths. CHIRON was looking for opportunities and the best recipe for such is to break the symmetry in the position. CHIRON did so adequately and all of a sudden, it was able to force a decision by sacrificing the exchange.

From Fig. 12a, White played 23. Rxf6. The resulting position was advantageous for CHIRON although no direct win was available. After some ten moves' reshuffling of pieces, CHIRON manage to organize a decisive attack. See Fig. 12b.

Black had returned the advantage of the exchange but was ensnared by a combination of mate threats and a passed pawn. CHIRON won soon after 33. Qh6 Rg8 34. dxe7 Qd7 35. Qh5 Qe8 36. Kh2 Nxe7 37. Nd6 Qf8 38. Nxf7+ Kg7 39. Qh6+ Kxf7 40. Rxf6+ Ke8 41. Rxf8+ Rxf8.

In the game GINKGO - SHREDDER the white player tried to keep its advantage from the opening and to build up a favourable position. However, SHREDDER was alert and found good places for its pieces resulting in the breaking open of the center when GINKGO gave too much attention on the king side, see Fig. 12c.

On the $\mathrm{d} 5$ square, many pieces were exchanged although this happened via a complex method of capturing, pinning and avoiding. After the battle of captures had cleared the air, an endgame of equal material with differently coloured bishops occurred. A draw was agreed.

KOMODO - JONNY played the English Four Knights' game. On the 9th and 11th moves, JONNY exchanged its bishop pair for a bishop and knight. That produced a slight inequality in the balance of material. Owing to the closed pawn formation, it could be argued that two knights are stronger in such a position than a knight and a bishop. From move 11 up to move 76 the programs kept all pawns on the board together with the remaining pieces, see Fig. 12d. Of course, the game was full of small tactics but all could be detected and refuted easily. After the first pawn exchange on move 77, there were more exchanges but ultimately, the balance was never disturbed in favor of one side or the other. On move 148 a draw was agreed. It was the longest game of the tournament.
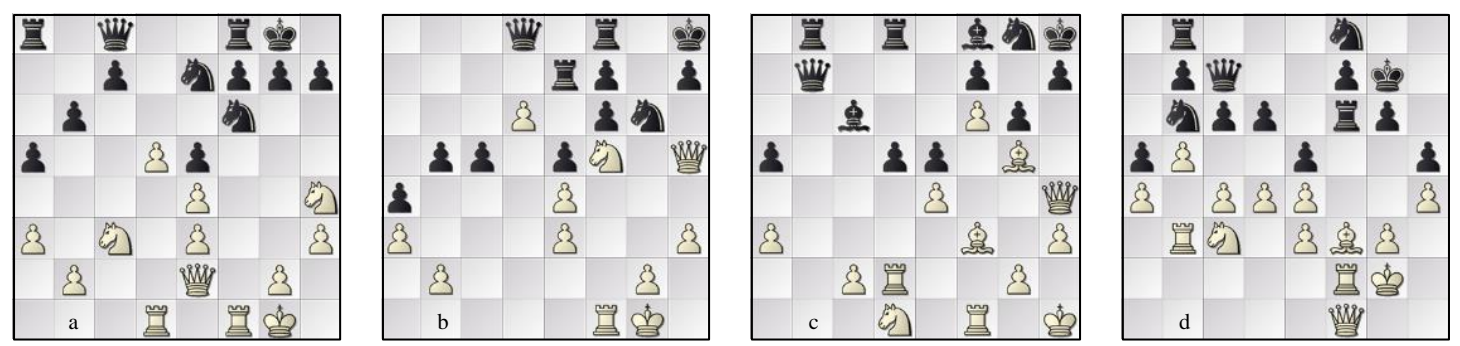

Fig. 12. Round 8: (a) CHIRON - THE BARON 23w and (b) 33w; (c) GINKGO - SHREDDER 27w, and (d) KOMODO - JONNY 77w.

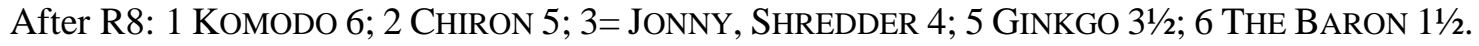




\section{Round 9: KOMODO - GINKGO 1-0, JONNY - CHIRON 1/2-1/2, SHREDDER - THE BARON 1-0.}

In this round, CHIRON had its last chance to set up the most exciting finale but it had to win. In the event, this chance was not taken as CHIRON drew and KOMODO won its game. Hence, KomODO became the official World Computer Chess Champion, 2019. Let us first honour KomODO by following its moves and seeing how it crushed GINKGO. After an opening of type KOMODO, namely avoiding specific opening lines, a characteristic position arose after 18 moves. KOMODO decided to play 20. Nd5 from Fig. 13a, which sacrificed a pawn for nothing more than open lines and the possession of strategic squares.

The battle continued and after 36. ... Ne6, see Fig. 13b, Komodo believed the time was ripe for a second pawn sacrifice by playing 37. a5. This bore fruit almost immediately. After 37. ... bxa5 38. bxa5 Qxa5 39. Qf5 Nd4 40. Qh5 Qc7, we arrived at the position of Fig. 13c.

White continued with 41. Bxh6 which guaranteed real advantage. Still, it was difficult to break down Black's position immediately and another ten moves were needed before, Fig. 13d, Black resigned, some 2.5 pawns down in the evaluation.

We are sure that many players would have continued fighting but not so GINKGO. In the continuation offered afterwards by STOCKFISH, it is clear that no escape was possible: 51... Kf8 52. Qxa5 Qxa5 53. Rxa5 Ne6 54. Ra7 Rc8 55. g4 Rb8 56. Rd1 Be7 57. Rda1 Re8 58. Rb1 1-0.

JONNY - CHIRON became a battle between them for second place. Their game was full of subtleties but the programs were too strong to become a victim of one of those subtleties. There was never a real chance for either side to gain an advantageous position. In a quiet battle, many pieces were exchanged and no territory was gained. After 48 moves, a draw was agreed.

SHREDDER - THE BARON was a Slav opening in which White allowed Black to take a pawn on b2 with its queen. That is always a dangerous manoeuver and SHREDDER showed why this is so. As the variation had been played earlier, the black player should have had deep knowledge of it in order to maintain its position on the board. As it went, White soon had, at the cost of a piece, a counter attack which later resulted in the regain of the material, see Fig. 13e.

Soon thereafter, White managed to get a passed a-pawn and a power position on Black's back ranks. The game ended on move 41 with win for SHREDDER.
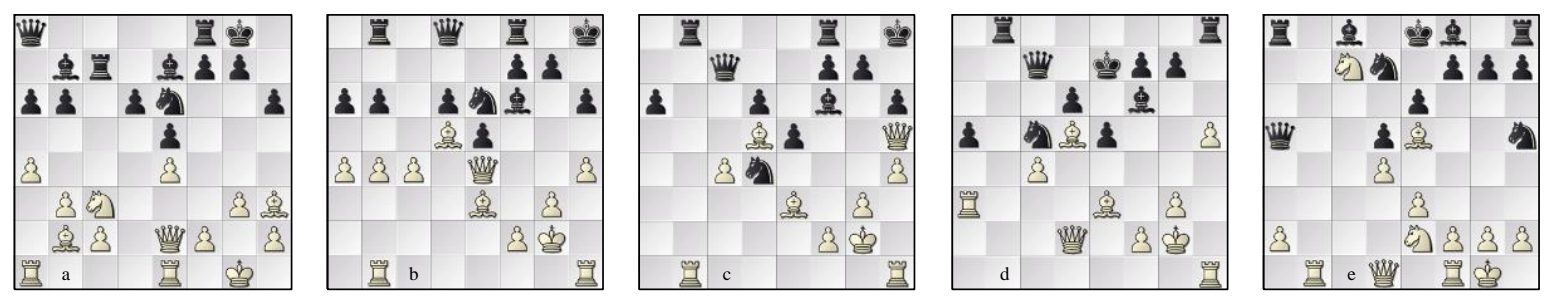

Fig. 13. Round 9: Komodo - Ginkgo (a) 20w, (b) 37w, (c) 41w and (d) 51b; (e) SHREDDER - THe BARON $15 \mathrm{~b}$.

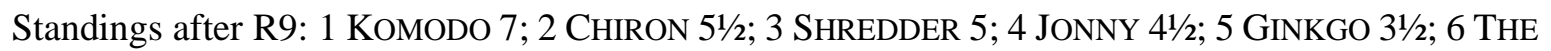
BARON $1 \frac{1}{2}$.

Round 10: GINKGO - THE BARON 1-0, SHREDDER - JONNY 1/2-1/2, CHIRON - KOMODO 1/2-1/2.

In the last round, CHIRON was unable to overcome KoMODO. Nevertheless, it made some attempts to defeat the new world champion. Number one and two of this championship played an exciting 
game which showed their equal playing strength. The first nine moves were the same as had been played in THE BARON - KOMODO, round 7. In that game, THE BARON played 10. Qf3 where CHIRON played 10. Qd2. Even after considering both games, it is not clear that Qd2 should be preferred. After that position, we saw an enormously unconventional fight in which both sides were in competition for the most remarkable move. It seems that a good candidate is move $\mathbf{2 4}$. $\mathbf{N f 7}$ played in the position of Fig. 14a.

Black replied with 24. ... Rb2, also a good candidate for the most remarkable move. In this Pandora's box of a game, many moves which were not considered to be the first choice seemed to be possible and even likely to deliver exciting outcomes. Play continued: 25. Bg5 Nb3+ 26. Kd1 Re8 27. Rxe8 Kxe8 28. Qe3+ Kxf7 29. Qe7+ Kg6 30. Qe8+ Qf7 31. h5+ 'CHIRON's contempt factor of - 0.33 avoiding the simplifying Queen exchange ..' Kg7 32. Bh6+ '.. twice' (Andrea), Fig. 14b. This result confirmed KOMODO as \#1 and CHIRON as \#2 in the WCCC 2019 Macau, China.

SHREDDER ended its tournament with a draw against JONNY, ensuring the last podium place for this former world champion. The game itself was full of content in which both programs showed that they were roughly of equal strength. For the spectator, SHREDDER had always a bit of an initiative but JONNY was never in danger. Therefore, the draw result was a fair result, rewarding the efforts of both sides.
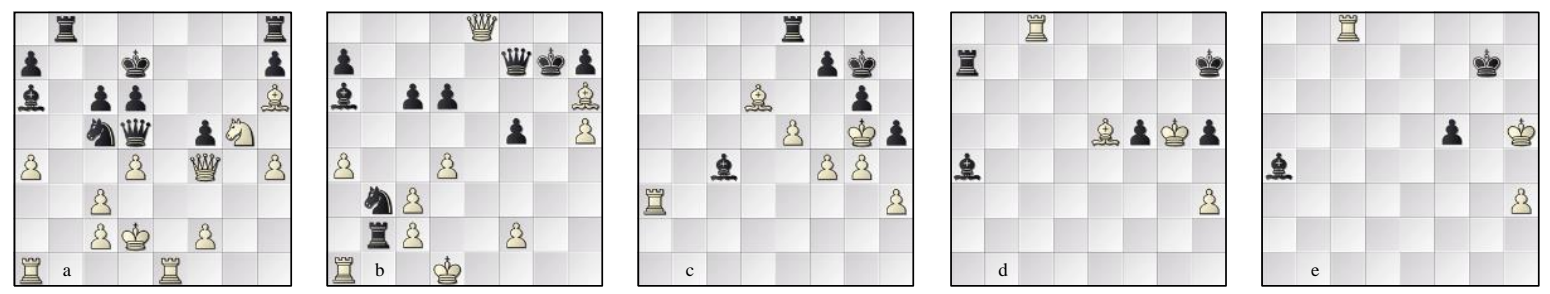

Fig. 14. Round 10: (a) CHIRON - KOMODO 24w and (b) 32b; (c) GINKGO - THE BARON 71b, (d) $81 \mathrm{~b}$ and (e) 83b.

Finally, we look at THE BARON. Although Richard Pijl saw its program losing its eighth game in this tournament, he could be happy with the 1.5 point scored against an ex-champion and a strong contender. The level of the current strongest chess programs of the world is impressive. In the last 30 years, there has been an enormous increase in average playing strength. Also, in this last game of WCCC 2019, THE BARON put up great resistance for 70 moves, see Fig. 14c, and only after the persistent efforts of GINKGO did it finally succumb on move 81, Fig. 14d, to a tricky and high calibre RB threat which led, Fig. 14e, to an actual, protracted R-vs-B mate in 35 moves.

Standings after R10: 1 KOMODO 71/2; 2 CHIRON 6; 3 SHREDDER 51/2; 4 JONNY 5; 5 GINKGO 41/2; 6 THE BARON $1 \frac{1}{2}$.

So ends the 2019 World Computer Chess Championship: once again, we salute the KoMODO team of authors - the late Don Dailey, Erdogan Günes, Larry Kaufman and Mark Lefler. The current champions defended their WCCC title with more positive and attacking chess, five wins, no losses. This may be a classic case of attack being the better form of defence.

We are approaching the $50^{\text {th }}$ anniversary of the ACM's first North American Computer Chess Championship. In that time, many engine authors - including past and current participants in the ICGA's events - have contributed to the state of the art in computer chess, an original AI challenge. Many of those ideas have been developed, fostered and demonstrated in these face-to-face meetings which the ICGA favours. Authors, organisers, fans and onlookers have regularly shared and enjoyed 
the ICGA's festival of computer chess, as witnessed by Figs. 15-16. It gives us particular pleasure to acknowledge the contributions of the WCCC 2019 participants and to thank them once again.

Tables 2-4 provide the full results in the various ways and the pgn file of the games (Krabbenbos et al., 2019a) includes annotations of many of the games with the decisive ones played out. It is easy to forget, when there are winners and losers, that all the engines here are at or beyond the top levels of human performance and that there is much to learn from any of these games.

Table 2

The WCCC 2019 cross-table

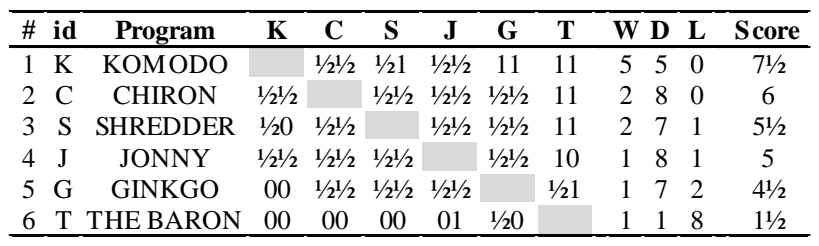

Table 3

The WCCC 2019 results, round by round

\begin{tabular}{|c|c|c|c|c|c|c|c|c|c|c|c|c|c|}
\hline \# & id & Program & r1 & $\mathbf{r} 2$ & r3 & r4 & r5 & r6 & r7 & r8 & r9 & r10 & Score \\
\hline 1 & $\mathrm{~K}$ & KOMODO & $\mathrm{Sb}^{1} / 2$ & Tw1 & $\mathrm{Jb}^{1} / 2$ & Gb1 & $\mathrm{CW}^{1 / 2}$ & Sw1 & Tb1 & $\mathrm{JW}^{1 / 2}$ & Gw1 & $\mathrm{Cb}^{1 / 2}$ & $71 / 2$ \\
\hline 2 & $\mathrm{C}$ & CHIRON & $\mathrm{Gw}^{1 / 2}$ & $\mathrm{Sw}^{1 / 2}$ & Tb1 & $\mathrm{JW}^{1 / 2}$ & $\mathrm{~Kb}^{1 / 2}$ & $\mathrm{~Gb}^{1 / 2}$ & $\mathrm{Sb}^{1 / 2}$ & Tw1 & $\mathrm{Jb}^{1} / 2$ & $\mathrm{Kw}^{1 / 2}$ & 6 \\
\hline 3 & S & SHREDDER & $\mathrm{Kw}^{1} 1 / 2$ & $\mathrm{Cb}^{1 / 2}$ & $\mathrm{Gw}^{1 / 2}$ & Tb1 & $\mathrm{Jb}^{1 / 2}$ & $\mathrm{~Kb} 0$ & $\mathrm{Cw}^{1 / 2}$ & $\mathrm{~Gb}^{1 / 2}$ & Tw1 & $\mathrm{JW}^{1 / 2}$ & $51 / 2$ \\
\hline 4 & $\mathrm{~J}$ & JONNY & Tb1 & $\mathrm{Gb}^{1 / 2}$ & $\mathrm{Kw}^{1 / 2}$ & $\mathrm{Cb}^{1 / 2}$ & $\mathrm{Sw}^{1 / 2}$ & Tw0 & $\mathrm{Gw}^{1 / 2}$ & $\mathrm{~Kb}^{1 / 2}$ & $\mathrm{Cw}^{1 / 2}$ & $\mathrm{Sb}^{1 / 2}$ & 5 \\
\hline 5 & G & GINKGO & $\mathrm{Cb}^{1 / 2}$ & $\mathrm{JW}^{1 / 2}$ & $\mathrm{Sb}^{1 / 2}$ & Kw0 & $\mathrm{Tb}^{1 / 2}$ & $\mathrm{Cw}^{1 / 2}$ & $\mathrm{Jb}^{1 / 2}$ & $\mathrm{Sw}^{1 / 2}$ & $\mathrm{~Kb} 0$ & Tw1 & $4^{1 / 2}$ \\
\hline 6 & $\mathrm{~T}$ & THE BARON & $\mathrm{J}_{\mathrm{w}} 0$ & $\mathrm{~Kb} 0$ & $\mathrm{Cw} 0$ & Sw0 & $\mathrm{Gw}^{1 / 2}$ & $\mathrm{Jb} 1$ & Kw0 & $\mathrm{Cb} 0$ & $\mathrm{Sb} 0$ & $\mathrm{Gb0}$ & $1 \frac{1 / 2}{2}$ \\
\hline
\end{tabular}

Table 4

WCCC 2019 progress, round by round

\begin{tabular}{cccccccccccc}
\hline$\#$ & Program & r1 & r2 & r3 & r4 & r5 & r6 & r7 & r8 & r9 & r10 \\
\hline 1 & KOMODO & $1 / 2$ & $11 / 2$ & 2 & 3 & $31 / 2$ & $41 / 2$ & $51 / 2$ & 6 & 7 & $71 / 2$ \\
2 & CHIRON & $1 / 2$ & 1 & 2 & $21 / 2$ & 3 & $31 / 2$ & 4 & 5 & $51 / 2$ & 6 \\
3 & SHREDDER & $1 / 2$ & 1 & $11 / 2$ & $21 / 2$ & 3 & 3 & $31 / 2$ & 4 & 5 & $51 / 2$ \\
4 & JONNY & 1 & $11 / 2$ & 2 & $21 / 2$ & 3 & 3 & $31 / 2$ & 4 & $41 / 2$ & 5 \\
5 & GINKGO & $1 / 2$ & 1 & $11 / 2$ & $11 / 2$ & 2 & $21 / 2$ & 3 & $31 / 2$ & $31 / 2$ & $41 / 2$ \\
6 & THE BARON & 0 & 0 & 0 & 0 & $1 / 2$ & $11 / 2$ & $11 / 2$ & $11 / 2$ & $11 / 2$ & $11 / 2$ \\
\hline
\end{tabular}

\section{REFERENCES}

CPW (2019). https://tinyurl.com/icga046. Biographies of programs, authors and operators.

Krabbenbos, J., van den Herik, H.J. and Haworth, G.McC. (2019a). WCCC 2019: the $25^{\text {th }}$ World Computer Chess Championship. http://centaur.reading.ac.uk/85845/: this note with annotated pgn files. ICGA Journal, 41(4), 206-221. doi: https://doi.org/10.3233/ICG-190125.

Krabbenbos, J., van den Herik, H.J. and Haworth, G.McC. (2019b). WCSC: the $9^{\text {th }}$ World Chess Software Championship. http://centaur.reading.ac.uk/85846/. ICGA Journal, 41(4), 222-236. doi: https://doi.org/10.3233/ICG-190126.

Krabbenbos, J., van den Herik, H.J. and Haworth, G.McC. (2019c). WSCC 2019: the 2019 World Speed Computer Chess Championship. http://centaur.reading.ac.u/85847/. ICGA Journal, 41(4), 237-240. doi: https://doi.org/10.3233/ICG-190127. 

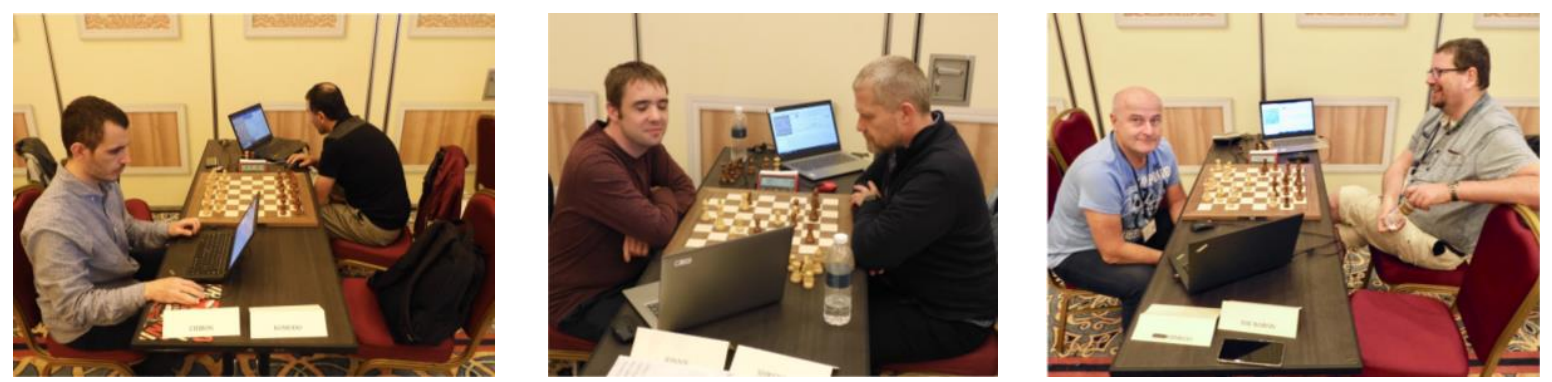

Fig. 15. (left to right) Ubaldo Andrea Farina, Erdogan Günes,

Johannes Zwanzger, Stefan Mayer-Kahlen, Wolfgang Zugrav and Richard Pijl.

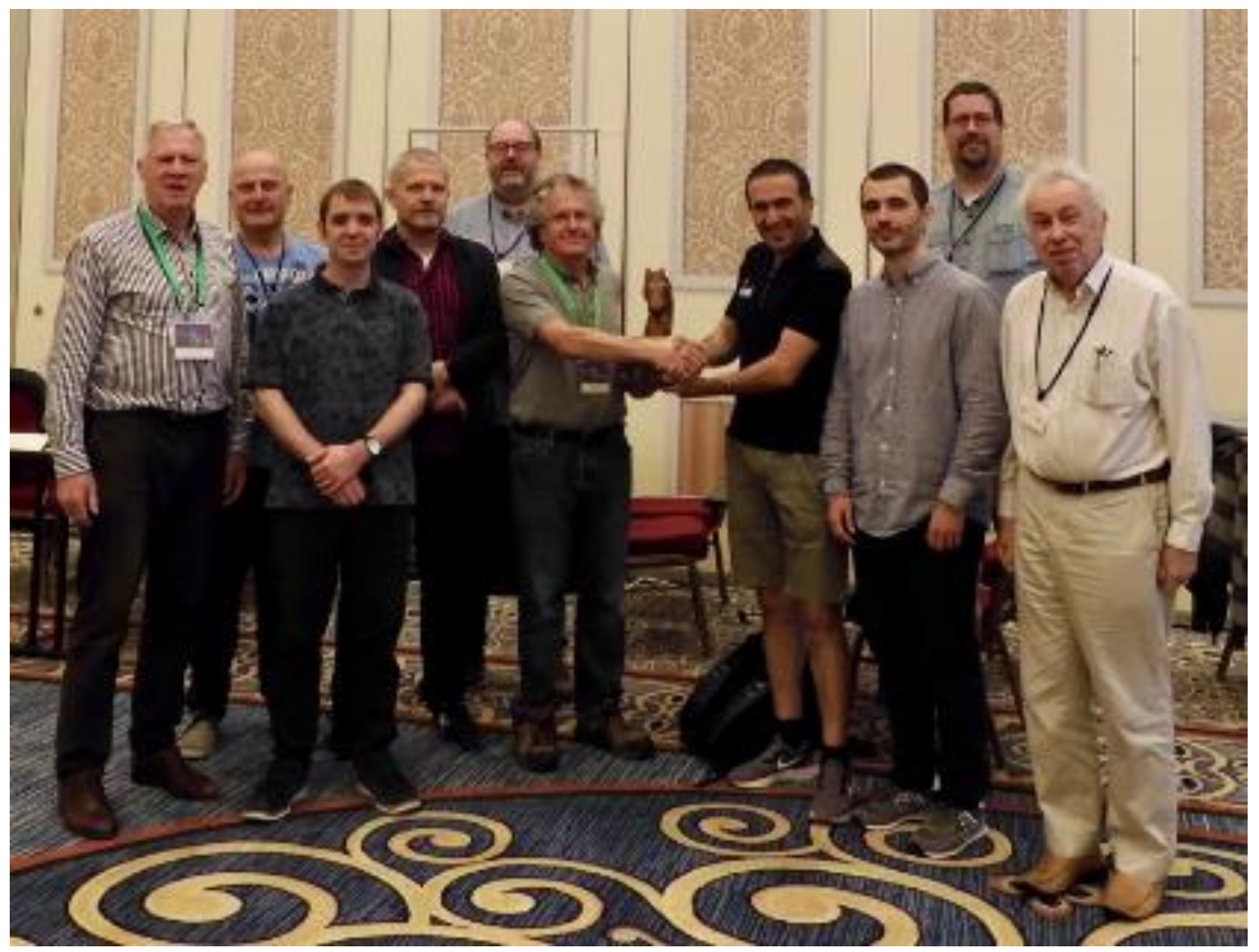

Fig. 16. The ICGA President, Jonathan Schaeffer presents the Shannon Trophy to Erdogan Günes for KomODO: (left to right) Jaap, Wofgang, Johannes, Stefan, Jan, Jonathan, Erdogan, Ubaldo, Richard and David. 